\title{
Antimicrobial activities of some marine Streptomycetes
}

Mohamed I. Abou-Dobara, Ahmed K.A. El-Sayed*, Amira A. El-Fallal, Gehan A. Zahran

Botany and Microbiology Department, Faculty of Science, Damietta University, Damietta, Egypt.

Received: 24 January 2016/ Accepted: 5 May 2016

* Corresponding author: akaelsayed@du.edu.eg

\begin{abstract}
Twenty marine actinomycetes were isolated from sediments and rhizosphere of some halophyte plants from coastal regions of North Delta, Egypt. Four isolates which showed a wide range of antimicrobial activities (inhibition for both Gram-positive and Gram-negative bacteria, and fungi) were selected and identified on the basis of their cellular morphology, physiological and chemotaxonomic characterization. The isolates were identified and named as Streptomyces albus strain DEG18, Streptomyces canaries strain REB9, Streptomyces sp strain REB5 and Streptomyces sp strain G12. Extraction of metabolites filtrate and biomass were carried out by ethyl acetate and acetone, respectively. In secondary screening, all four Streptomyces strains showed antibacterial activity against Enterobacter cloaca and antifungal activity toward Fusarium oxysporum, three strains out of them showed antibacterial activity against Bacillus cereus, whereas two showed activity against Bacillus subtilis and Staphylococcus aureus, whereas some strains showed activity toward Klebsiella pneumoniae and Alternaria alternata.
\end{abstract}

Keywords: marine Streptomyces, antimicrobial activity.

\section{Introduction}

The continuous search for new antimicrobial compounds or new microorganism strains producing antimicrobial agents becomes necessary because of the increase of antibiotic resistant pathogens and toxicity of some chemical antibiotics. Marine biotechnology environment has opened up unexpected new horizons for finding novel organisms for trapping their potential resources (Ravenschlay et al., 1999; Stach et al., 2003; Jensen et al., 2005; Lam, 2006). However, culturally independent methods have demonstrated that marine sediments contain a wide range of unique microorganisms. Actinomycetes have a profound role in the marine environment apart from antibiotic production (Das et al., 2006). Actinomycetes are aerobic, spore forming gram positive bacteria, characterized by substrate and aerial mycelia growth (Lechevalier and Lechevalier, 1981). Actinomycetes are the most economically and biotechnologically valuable microorganisms due to their potential in antimicrobial activity. They have produced a wide range of secondary metabolites of various medical importance such as antibiotics, antagonistic agents, including antibacterials, antifungals, antiprotozoans as well as antivirals, pharmacological agents, including antitumorals, immunomodulators, neurological agents and enzyme inhibitors, agrobiologicals, including insecticides, pesticides and herbicides, and compounds with regulatory activities, such as growth factors, siderophores or morphogenic 
agents and immunosuppressant (Adegboye and Bablola, 2013). A large number of antibiotics were obtained and reported from the members of the genus Streptomyces only (Alan and James, 2007; Lyudmila et al., 2008; Junker et al., 2009; Koch and Loffler, 2009; Hotam et al., 2013). Pharmacological and agricultural screens are increasingly being used in combination with antimicrobial tests, to detect simultaneous bioactivities for a given compound. This has revealed several novel therapeutic and agrobiological agents and previously unknown biological activities for antibiotics (Berdy, 2005). Actinomycetes in marine environments are often under extreme conditions of temperature, pressure, salinity and depletion of micronutrients, with survival and proliferation often depending on their ability to produce biologically active compounds (Bull et al., 2000). It is believed that marine actinomycetes may have different characteristics from terrestrial actinomycetes and therefore might produce novel bioactive metabolites and new antibiotics (Ramesh and Mathivanan, 2009; Hames-Kocabas and Uzel, 2012), so, marine actinomycetes have attracted great attention to search novel antibiotics derived from new microorganisms (Carte, 1996; Kijjoa and Sawangwong, 2004). The research to date supports this hypothesis and it has been shown that marine actinomycetes produce novel types of new secondary metabolites (Lam 2006; Fenical and Jensen, 2006). Many of these metabolites possess novel biological activities and have the potential to be developed as therapeutic agents (Feling et al., 2003; Maldonado et al., 2005). However, this work aimed to isolate and identify antimicrobial producing marine actinomycetes as a potential source for production of antimicrobial agents.

\section{Materials and Methods}

\section{Collection of Samples}

Soil samples were collected at a depth of 10-20 cm from sediments of several different sites from coastal regions of North Delta, Egypt. Some samples were isolated from plant rhizosphere and a mucilaginous layer of algae that are grown on marine rocks. The collection sites and locations of the sampling were Ras El-Bar, El-Sheikh Dergham, Manzala Lake bank, Damietta ElGededa and Gamasa. Physical properties of water samples, including $\mathrm{pH}$, total dissolved salts (TDS) and electric conductivity (EC) were recorded.

\section{Isolation of actinomycetes}

Two gm of samples or five parts of one inch plant root samples included soil particles were added in $18 \mathrm{ml}$ of sterile sea water, vortexed and diluted with sterile sea water as in dilution agar plating method (Johnson et al., 1959). Aliquots (150ul) of each dilution were respectively spread on the surface of the starch casein agar medium (starch $10 \mathrm{gm}$; casein $2 \mathrm{gm} ; \mathrm{NaCl} 6 \mathrm{gm} ; \mathrm{KH}_{2} \mathrm{PO}_{4} 0.5 \mathrm{gm}$; $\mathrm{MgSO}_{4} 0.5 \mathrm{gm}$; agar $18 \mathrm{gm}$ and sterile sea water $1000 \mathrm{ml})$. The $\mathrm{pH}$ was adjusted to $7.2-7.4$ prior to autoclaving with the addition of sterile nalidixic acid and cycloheximide at $10 \mu \mathrm{g} / \mathrm{ml}$ and $20 \mu \mathrm{g} / \mathrm{ml}$, respectively to diminish the growth of marine bacteria and fungi. The plates were incubated at $28 \pm 2^{\circ} \mathrm{C}$ for 14 - 21 days. The purified actinomycetes were preserved on starch-casein agar slopes at $4{ }^{\circ} \mathrm{C}$ and in glycerol $(40 \% \mathrm{v} / \mathrm{v})$ at $80^{\circ} \mathrm{C}$ for longer storage periods.

\section{Identification of the most potential actinomycetes}

\section{- Morphological and physiological characteristics}

After selection of actinomycetes isolates according to their antagonistic and antimicrobial activities, their identification was carried out by studying their morphological, cultural and physiological characteristics. Streptomycetes species used in this investigation was identified according to the International Streptomyces Project (ISP) (Shirling and Gottlieb, 1968a; 1968b; 1969; 1972; Pridham and Tresner, 1974a; 1974b; Bergey's Manual of Systematic Bacteriology (Williams et al., 1989). Their morphological characters such as colony characteristics, type of aerial hyphae, their branching, growth of vegetative hyphae and spore formation were examined by light microscope and by JSM-5300, Jed Scanning electron microscope at Alexandria University. The physiological characteristics included gel liquefaction, utilization of starch, coagulation of milk, decomposition of cellulose and utilization of sugar.

\section{- Cultural characters}

Determination of the actinomycetes isolates colour; the colour of growth, sporulation aerial hyphae, substrate hyphae and diffusion of their pigment into the media were assayed on different media such as: starch casein agar, starch nitrate 
agar, starch-ammonium sulphate agar, CzapekDox agar, glycerol - asparagine agar, glycerol yeast agar and CM-1 agar media.

\section{- Chemotaxonomic analysis of the selected Streptomyces isolates}

Determination of the cell wall composition, including diaminopimelic acid (DAP) isomers and sugars was based upon the methods of Becker et al. (1964; 1965), Stanek and Roberts (1974).

Bioactivity of isolates in primary screening

The antimicrobial activities of the isolated actinomycetes were detected against six local bacteria: Enterobacter cloacae, Klebsiella pneumoniae and Escherichia coli (Gram-negative bacteria) Bacillus cereus, $B$. subtilis and Staphylococcus aureus (Gram-positive bacteria) and four local fungi: Fusarium oxysporum, Aspergillus niger, Aspergillus flavus and Alternaria alternata by using diffusion method.

\section{- Screening on solid media}

The streptomycetes isolates were grown on starch casein agar media for 10 days at $28 \pm 2^{\circ} \mathrm{C}$. By using an agar plate diffusion method (Wu, 1984), agar discs were cut off by a sterilized cork-borer ( $1 \mathrm{~cm}$ diameter) and transferred into the surface of agar plates previously inoculated with tested microorganisms. The bacteria were grown on nutrient agar while fungi were grown on potato dextrose agar media. The antagonistic activity was determined by measuring the inhibition zone diameter $(\mathrm{mm})$.

\section{- Screening using liquid media}

The isolates were grown on starch casein broth and adjusted the $\mathrm{pH}$ to 7.2- 7.4. The cultures were incubated on a rotary shaker $(150 \mathrm{rpm})$ at $28 \pm 2^{\circ} \mathrm{C}$ for 9 days. Metabolites were centrifuged and the supernatants were filtered by using sterilized $45 \mu \mathrm{m}$ Millipore filter paper. By using sterile corkborer (1 $\mathrm{cm}$ diameter), the hollow pores in inoculated nutrient agar and potato dextrose agar (PDA) were made and filled with $300 \mu 1$ of cellfree supernatant for each pore. The antimicrobial activities were assayed by measuring the inhibition zones diameter $(\mathrm{mm})$ of bacteria and fungi.

\section{Bioactivity of strains in secondary screening}

Agar diffusion method was used to determine the antibacterial and antifungal activity of the ethyl acetate extract of metabolite filtrates and biomass acetone extracts as inhibition zone (mm). This was compared with the crude metabolites as described above.

\section{Extraction of antimicrobial agents from metabolites and biomass}

Extraction of antimicrobial agents from metabolites and biomass of most potential actinomycetes were carried out by growing them on a starch casein broth. The medium was adjusted to $\mathrm{pH} 7.2 \pm 0.2$ by $1 \mathrm{~N} \mathrm{NaOH}$ and $1 \mathrm{~N} \mathrm{HCl}$, distributed into $250 \mathrm{ml}$ conical flask containing 50 $\mathrm{ml}$ and inoculated using spore suspensions. Flasks were incubated at $28^{\circ} \pm 2^{\circ} \mathrm{C}$ for 9 days on rotaryshaker at $150 \mathrm{rpm}$. After fermentation, the antimicrobial compounds were extracted by using ethyl acetate. The culture broth was centrifuged at $5,000 \mathrm{rpm}$ for 10 minutes and filtered to remove biomass. The cell-free supernatant was transferred to a separating flask. Ethyl acetate was added with a ratio of $1: 1(\mathrm{v} / \mathrm{v})$ and shaken vigorously for 10 minutes. The top layer is transferred to a clean glass tube. Ethyl acetate extraction was done twice. The supernatant was collected and passed throughout a column containing traces of sodium sulphate and the filtrate was evaporated to dryness. One mg dry extract were dissolved in $10 \mathrm{ml}$ of methanol and the antimicrobial activity was bioassayed using only $200 \mu \mathrm{l}$. Acetone was added for biomass and shaken vigorously for 10 minutes. The top layer of the extract is transferred to a clean glass tube. The antimicrobial activity

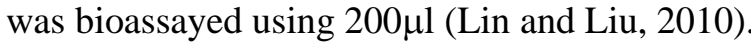

\section{Results and Discussion}

Twenty marine actinomycetes species were isolated from different marine sites in coastal regions of North Delta, Egypt within the year of 2011 (Table 1). Some marine actinomycetes were too difficult to be isolated from some other sites. Despite of many efforts of scientists to success the marine actinomycetes isolation, their abundance and diversity are still rare (Stach et al., 2003; Maldonado et al., 2005; Gontang et al., 2007; Bouvier and del Giorgio, 2007). Most of the marine actinomycetes live in sea water in the form of few colonies and cannot grow under laboratory 
conditions (Manivasagan et al., 2014). The media containing macromolecules like casein and supplemented with sea water are suitable for promoting the growth of rare marine actinomycetes (Qiu et al. 2008; Bredholdt et al. 2008; Hong et al. 2009; Zhang and Zhang, 2011). All the purified isolates showed morphological characteristics of typical Streptomyces species, as their colonies possessed an earthy odor and were slow growing, aerobic, powdery, folded with aerial and substrate mycelia of different colors (Anderson and Wellington, 2001).

Morphological studies were carried out and the characteristics of the isolates were compared with the standard characteristics described in Bergey's manual of systematic Bacteriology (William et al., 1989). They formed colored, tough and leathery colonies that were hard to pick from the culture media. Microscopic studies also showed that the cell of isolates formed long branched network of mycelia which is characteristic of Streptomyces sp as previously described by Kieser et al. (2000). These isolates were categorized culturally and morphologically into two series according to the color of their mature sporulating aerial mycelium. Ten out of them were grouped in the grey color group and the other were belonging to the white color group as shown in Table (1). Estimation of the $\mathrm{pH}$, total dissolved salts (TDS) and electric conductivity (EC) for the collection locations were useful to optimize the suitable growth conditions for the isolates. The measured $\mathrm{pH}, \mathrm{EC}$ and TDS were about $7.0 \pm 0.4,54 \mathrm{~ms} / \mathrm{cm}$ and $34.560 \mathrm{~g} / \mathrm{l}$, respectively, for most sites.

Table 1. Collection sites of soil samples and color grouping of the isolates.

\begin{tabular}{|c|c|c|c|c|c|c|c|c|c|c|c|}
\hline $\begin{array}{c}\text { Color of } \\
\text { series }\end{array}$ & $\begin{array}{c}\text { Isolate } \\
\text { No }\end{array}$ & $\begin{array}{l}\text { Color of } \\
\text { aerial } \\
\text { mycelia }\end{array}$ & $\begin{array}{c}\text { Color of } \\
\text { substrate } \\
\text { mycelia }\end{array}$ & Cover plant & $\begin{array}{c}\text { Site of } \\
\text { sampling }\end{array}$ & $\begin{array}{c}\text { Color } \\
\text { of series }\end{array} \mid$ & $\begin{array}{l}\text { Isolate } \\
\text { No. }\end{array}$ & $\begin{array}{c}\text { Color of } \\
\text { aerial } \\
\text { mycelia }\end{array}$ & $\begin{array}{c}\text { Color of } \\
\text { substrate } \\
\text { mycelia }\end{array}$ & Cover plant & $\begin{array}{c}\text { Site of } \\
\text { sampling }\end{array}$ \\
\hline \multirow{10}{*}{ Grey } & 1 & Gray & Grey & $\begin{array}{l}\text { Mucilaginous algal } \\
\text { layer }\end{array}$ & Ras Bar & \multirow{10}{*}{ White } & 10 & White & White & Sea shore & Gamasa \\
\hline & 2 & Gray & Brown & $\begin{array}{l}\text { Mucilaginous algal } \\
\text { layer }\end{array}$ & Ras Bar & & 11 & White & White & Sea shore & Gamasa \\
\hline & 3 & Gray & Brown & $\begin{array}{l}\text { Rhizosphere of } \\
\text { Salsola kali }\end{array}$ & Ras Bar & & 12 & White & White & Sea shore & Gamasa \\
\hline & 4 & Gray & Brown & \begin{tabular}{|l|} 
Rhizosphere \\
Salsola kali \\
\end{tabular} & Ras Bar & & 13 & White & White & Sea shore & Gamasa \\
\hline & 5 & $\begin{array}{l}\text { Light } \\
\text { gray }\end{array}$ & $\begin{array}{l}\text { Light } \\
\text { grey }\end{array}$ & $\begin{array}{l}\text { Rhizosphere } \\
\text { Spergularia } \\
\text { marina }\end{array}$ & Ras Bar & & 14 & White & white & \begin{tabular}{|l|} 
Rhizosphere \\
of $\quad$ Bassia \\
indica
\end{tabular} & $\begin{array}{l}\text { Manzala } \\
\text { lake bank }\end{array}$ \\
\hline & 6 & Gray & Brown & $\begin{array}{l}\text { Sediment around } \\
\text { Spergularia } \\
\text { marina } \\
\end{array}$ & Ras Bar & & 15 & White & White & \begin{tabular}{|l|} 
Rhizosphere \\
of $\quad$ Bassia \\
indica
\end{tabular} & $\begin{array}{l}\text { Manzala } \\
\text { lake bank }\end{array}$ \\
\hline & 7 & Gray & $\begin{array}{l}\text { Light } \\
\text { grey }\end{array}$ & $\begin{array}{l}\text { Sediment around } \\
\text { Spergularia } \\
\text { marina }\end{array}$ & Ras Bar & & 17 & White & white & $\begin{array}{l}\text { Rizosphere } \\
\text { of } \\
\text { Zygophyllum } \\
\text { album }\end{array}$ & $\begin{array}{l}\text { El- } \\
\text { Gamail } \\
\text { beach }\end{array}$ \\
\hline & 8 & Gray & $\begin{array}{l}\text { Light } \\
\text { grey }\end{array}$ & $\begin{array}{l}\text { Sediment around } \\
\text { S. marina }\end{array}$ & Ras Bar & & 18 & White & White & $\begin{array}{l}\text { Rhizosphere } \\
\text { of } C \text {. murale } \\
\end{array}$ & \begin{tabular}{|l|} 
Damietta \\
Gededa \\
\end{tabular} \\
\hline & 9 & Gray & yellow & $\begin{array}{l}\text { Mucilaginous algal } \\
\text { layer }\end{array}$ & Ras Bar & & 19 & White & White & \begin{tabular}{|l|} 
Rhizosphere \\
of \\
Ceratophyllu \\
$m$ demersum
\end{tabular} & $\begin{array}{l}\text { Manzala } \\
\text { lake bank }\end{array}$ \\
\hline & 16 & Gray & Brown & $\begin{array}{l}\text { Rhizosphere } \\
\text { Halocnemum } \\
\text { strobilaceum }\end{array}$ & $\begin{array}{l}\text { Manzala } \\
\text { lake bank }\end{array}$ & & 20 & White & White & $\begin{array}{l}\text { Rhizosphere } \\
\text { of } \\
\text { Ceratophyllu } \\
\text { m demersum }\end{array}$ & $\begin{array}{l}\text { Manzala } \\
\text { lake bank }\end{array}$ \\
\hline
\end{tabular}

\section{Bioactivity of actinomycete isolates in primary screening}

The primary screening exhibited that all twenty actinomycete isolates tested did not have bioactive metabolites against Aspergillus niger and Alternaria alternata. The actinomycete isolates from different color groups displayed varying degree of inhibition of the tested bacteria and fungi. Isolates coded by: REB5, REB9, G12 and DEG 18 exhibited a significant and wide range of antimicrobial activities against Enterobacter cloacae (Gram-negative bacterium), Bacillus cereus and Bacillus subtilis (Gram-positive bacteria) followed closely by Staphylococcus aureus that was inhibited by three out of four $(75 \%)$ isolates tested. Klebsiella pneumoniae and Escherichia coli (25\%) were the less sensitive pathogens tested. In addition to the antifungal activities of three out four $(75 \%)$, isolates were 
active against Fusarium oxysporum as shown in Table (2). The antibacterial pattern exhibited by the strains in the present investigation, where the antagonism against Gram-positive bacteria was greater than Gram-negative one and was almost similar to those the ones reported by Tan et al. (2004) and Kavithambigai (2006). The reason for different sensitivity between Gram-positive and Gram-negative bacteria could be due to the morphological differences between these microorganisms; Gram-negative bacteria having an outer polysaccharide membrane carrying the structural lipopolysaccharide components. This makes the cell wall impermeable to lipophilic solutes. Gram-positive bacteria were more susceptible to having only an outer peptidoglycan layer, which was not an effective permeability barrier (Pandey et al., 2002). The most potent isolates were selected for more study and identification. Shams et al. (2015) isolated some marine actinomycete isolates from Lipar area of Oman Sea and showed a good antibacterial activity against Staphylococcus species than Gram-negative bacteria including Escherichia coli.

Table 2. Inhibition spectrum $(\mathrm{mm})$ of four actinomycetes isolates against the test pathogens in primary screening method. Data represented as means of three replications with standard error.

\begin{tabular}{|l|c|c|c|c|c|c|c|c|c|c|}
\hline \multirow{2}{*}{ Isolate code } & \multicolumn{9}{|c|}{ Tested bacteria } & \multicolumn{4}{c|}{ Tested fungi } \\
& $\begin{array}{c}\text { E. } \\
\text { cloacae }\end{array}$ & K. penumoniae & $\begin{array}{c}E . \\
\text { coli }\end{array}$ & $\begin{array}{c}\text { S. } \\
\text { aureus }\end{array}$ & $\begin{array}{c}\text { B. } \\
\text { cereus }\end{array}$ & $\begin{array}{c}\text { B. } \\
\text { subtilis }\end{array}$ & $\begin{array}{c}\text { F. } \\
\text { oxysporium }\end{array}$ & $\begin{array}{c}\text { A. } \\
\text { niger }\end{array}$ & $\begin{array}{c}\text { A. } \\
\text { Flavus }\end{array}$ & A. alternata \\
\hline REB5 & $6.27 \pm 0.17$ & 0.0 & $3.33 \pm 0.09$ & $5.3 \pm 0.15$ & $4.6 \pm 0.21$ & $2.37 \pm 0.1$ & $4.3 \pm 0.44$ & 0.0 & 0.0 & 0.0 \\
\hline REB9 & $10.5 \pm 0.29$ & 0.0 & 0.0 & $0.73 \pm 0.7$ & $2.8 \pm 0.17$ & $2.75 \pm 0.14$ & $4.5 \pm 0.29$ & 0.0 & 0.0 & 0.0 \\
\hline G12 & $5.0 \pm 0.29$ & 0.0 & 0.0 & 0.0 & $6.67 \pm 0.44$ & $6.1 \pm 0.1$ & $7.3 \pm 0.44$ & 0.0 & $1.5 \pm 0.29$ & 0.0 \\
\hline DEG18 & $15.4 \pm 1.45$ & $4.1 \pm 0.81$ & 0.0 & $6.5 \pm 0.3$ & $5.0 \pm 0.29$ & $1.3 \pm 0.17$ & 0.0 & 0.0 & 0.0 & 0.0 \\
\hline
\end{tabular}

\section{Identification of the most potential actinomycetes}

Examination of four grown isolates on starch casein medium at $28^{\circ} \pm 2^{\circ} \mathrm{C}$ for 7 days under light microscope and JSM-5300, Jed Scanning electron microscope revealed that, only the isolate REB5 has a spiral sporophore and spiny spore surface, however, isolates REB9, G12 and DEG18 have spiral sporophore and smooth spore surface as shown in Figure (1). The substrate mycelium (SM) had no distinctive color. It varied depending on the type of the used media. Strains grew well to moderate on the tested organic and synthetic media. The color of aerial mycelium varied depending on the type of used media (Table 3 ).

The results of the chemotaxonomic and physiological experiments of selected strains are shown in Table 4. It can be seen that the presence of a chemotype I cell wall characterized by L-DAP and no characteristic sugars were detected. All isolates can coagulate the milk and cannot produce melanoid pigments. All selected isolates can grow on starch casein media with $60 \mathrm{~g} / \mathrm{l}$ of salt concentration, whereas strain G12 can grow up to $70 \mathrm{~g} / \mathrm{l}$ of salt concentration, which shows the salinity tolerance ability as the characteristics of marine microorganisms.

The best media for growth of all these actinomycete isolates were starch casein agar media It was found that starch casein media are quite suitable and used for isolation of marine actinomycetes, this result agreed with the results of Lin and Liu (2010); Ballav et al., (2015). The isolate REB5 was additionally preferred to grow on starch nitrate agar and Czapek-Dox agar, while isolate DEG18 was additionally able to grow on starch nitrate agar; this result was in agreement with Attimarad et al. (2012) who reported that starch nitrate agar media were suitable for production of bioactive metabolites from some marine Streptomyces species against

The color of aerial mycelium was grey for isolate REB9 with yellow diffusible pigments, and isolate REB5 without diffusible pigments. It was off white for the isolates G12 and DEG18 on the studied media (Table 3). All the pigmentations of the studied isolates were non-sensitive towards the $\mathrm{HCl}$ and $\mathrm{NaOH}$ except isolate REB9 which showed sensitivity with $\mathrm{HCl}$. The isolates REB5, REB9, G12 and DEG18 were able to grow at a wide range of temperatures $\left(8^{\circ} \mathrm{C}\right.$ to $\left.45^{\circ} \mathrm{C}\right)$. The optimum growth was at $28^{\circ} \mathrm{C} \pm 2{ }^{\circ} \mathrm{C}$. The $\mathrm{pH} 6,7$, and 8 were suitable for their growth with optimum $\mathrm{pH}$ at 7. According to Guimarães et al. (2004), the $\mathrm{pH}$ of the culture medium is one of the most important environmental factors, because it exerts a marked effect on the activity of several enzymes that catalyze metabolic reactions, as well as exerting significant influence on complex physiological phenomena such as membrane 
permeability and cell morphology (Guimarães $e t$ al., 2004). Bundale et al. (2015) reported that $\mathrm{pH}$ 7 was found to be optimum for both growth as well as bioactive metabolite production from isolate $\mathrm{R} 3$ toward Bacillus cereus. The characteristics of four strains such as obtained cellular morphology, cultural properties, physiological and chemotaxonomic characterization were compared with those of known species of actinomycetes described in Bergey's Manual of Systematic Bacteriology (Williams et al., 1989), suggested strongly that these isolates belongs to genus Streptomyces and named as Streptomyces sp strain REB5, Streptomyces canaries strain REB9, Streptomyces sp strain G12 and Streptomyces albus strain DEG18. They were isolated from three sites (Ras El-Bar, Gamasa and Damietta ElGededa).
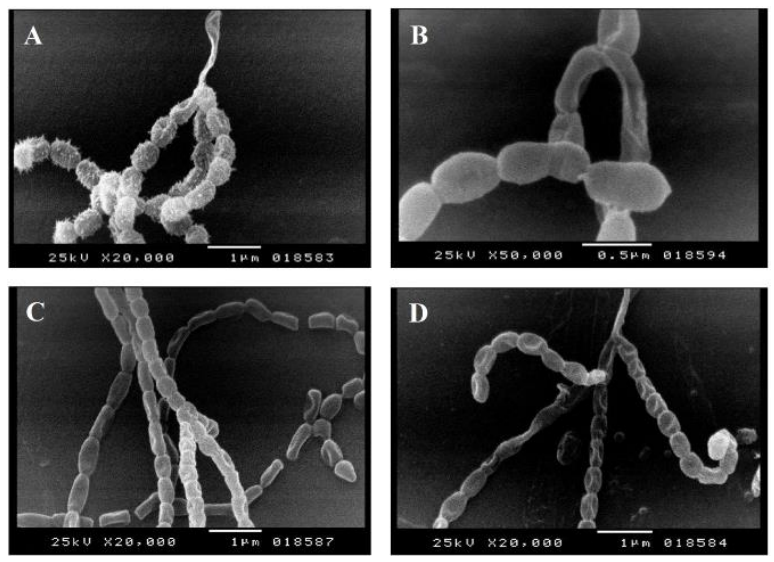

Figure 1. Spore and sporophore morphology of Streptomyces isolates using JSM-5300, Jed Scanning electron microscope. A: Streptomyces sp strain REB5, B: Streptomyces albus strain DEG18, C: Streptomyces sp strain G12, D: Streptomyces canaries strain REB9.

Staphylococcus aureus, Bacillus subtilis and Escherichia coli.

Table 3. Cultural characteristics of the selected Streptomyces strains.

\begin{tabular}{|c|c|c|c|c|c|c|c|c|c|}
\hline \multicolumn{2}{|c|}{ Characters } & Strain code & $\begin{array}{l}\text { Starch } \\
\text { casein }\end{array}$ & Starch nitrate & $\begin{array}{c}\text { Starch amm. } \\
\text { sulphate }\end{array}$ & $\begin{array}{l}\text { Czapex- } \\
\text { Dox }\end{array}$ & CM-1 & $\begin{array}{c}\text { Glycerol- } \\
\text { Yeast }\end{array}$ & $\begin{array}{c}\text { Glycerol- } \\
\text { Asparagine }\end{array}$ \\
\hline \multirow{4}{*}{\multicolumn{2}{|c|}{ 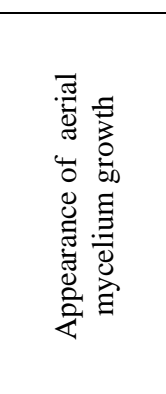 }} & REB5 & $\begin{array}{l}\text { Powdery, } \\
\text { good }\end{array}$ & $\begin{array}{l}\text { Powdery, } \\
\text { good }\end{array}$ & $\begin{array}{l}\text { Powdery, } \\
\text { very weak }\end{array}$ & $\begin{array}{l}\text { Powdery, } \\
\text { good }\end{array}$ & $\begin{array}{l}\text { Powdery, } \\
\text { good }\end{array}$ & $\begin{array}{l}\text { Powdery, } \\
\text { weak }\end{array}$ & $\begin{array}{c}\text { Powdery, very } \\
\text { weak }\end{array}$ \\
\hline & & REB9 & $\begin{array}{l}\text { Powdery, } \\
\text { good }\end{array}$ & $\begin{array}{l}\text { Powdery, } \\
\text { good }\end{array}$ & $\begin{array}{l}\text { Powdery, } \\
\text { very weak }\end{array}$ & $\begin{array}{l}\text { Powdery, } \\
\text { good }\end{array}$ & $\begin{array}{l}\text { Powdery, } \\
\text { good }\end{array}$ & $\begin{array}{c}\text { Powdery, } \\
\text { weak }\end{array}$ & $\begin{array}{l}\text { Powdery, } \\
\text { moderate }\end{array}$ \\
\hline & & G12 & $\begin{array}{l}\text { Powdery, } \\
\text { good }\end{array}$ & $\begin{array}{l}\text { Powdery, no } \\
\text { aerial growth }\end{array}$ & $\begin{array}{c}\text { Powdery, } \\
\text { moderate } \\
\text { aerial growth }\end{array}$ & $\begin{array}{l}\text { Powdery, } \\
\text { good }\end{array}$ & $\begin{array}{l}\text { Powdery, } \\
\text { good }\end{array}$ & $\begin{array}{l}\text { Powdery, no } \\
\text { aerial growth }\end{array}$ & $\begin{array}{l}\text { Powdery, no } \\
\text { aerial growth }\end{array}$ \\
\hline & & DEG18 & $\begin{array}{l}\text { Powdery, } \\
\text { good }\end{array}$ & $\begin{array}{l}\text { Powdery, no } \\
\text { aerial growth }\end{array}$ & $\begin{array}{c}\text { Powdery, } \\
\text { moderate } \\
\text { aerial growth }\end{array}$ & $\begin{array}{l}\text { Powdery, } \\
\text { good }\end{array}$ & $\begin{array}{l}\text { Powdery, } \\
\text { good }\end{array}$ & $\begin{array}{l}\text { Powdery, no } \\
\text { aerial growth }\end{array}$ & $\begin{array}{l}\text { Powdery, no } \\
\text { aerial growth }\end{array}$ \\
\hline \multirow{12}{*}{$\begin{array}{l}4 \\
\dot{0} \\
\dot{0}\end{array}$} & \multirow{4}{*}{ 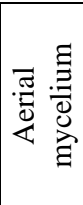 } & REB5 & Grey & Grey & Pale yellow & $\begin{array}{l}\text { Whitish } \\
\text { grey }\end{array}$ & Orange grey & Pale creamy & White \\
\hline & & REB9 & Grey & White & Non & Grey & White & White & Whitish grey \\
\hline & & G12 & Off white & Non & Off white & Pale yellow & White & Non & Non \\
\hline & & DEG18 & Off white & White & Off white & Pale yellow & White & Non & Non \\
\hline & \multirow{4}{*}{ 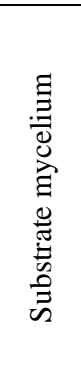 } & REB5 & Pale grey & Dark grey & Yellow & White grey & Brown & Off white & Off white \\
\hline & & REB9 & yellow & Off white & Yellow & $\begin{array}{c}\text { Pale } \\
\text { Yellowish } \\
\text { grey }\end{array}$ & $\begin{array}{l}\text { Yellowish } \\
\text { grey }\end{array}$ & Yellow & Brown \\
\hline & & G12 & Off white & Off white & Off white & $\begin{array}{c}\text { Pale } \\
\text { yellowish } \\
\text { green }\end{array}$ & Pale yellow & Pale yellow & Pale yellow \\
\hline & & DEG18 & Off white & Off white & Off white & $\begin{array}{c}\text { Pale } \\
\text { yellowish } \\
\text { green } \\
\end{array}$ & Pale yellow & Pale yellow & Pale yellow \\
\hline & \multirow{4}{*}{ 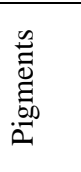 } & REB5 & Grey & Non & Non & Non & Non & Non & Non \\
\hline & & REB9 & Yellow & Non & Non & Pale yellow & Dark yellow & Non & Brown \\
\hline & & G12 & Non & Non & Non & Non & Non & Non & Non \\
\hline & & DEG18 & Non & Non & Non & Non & Non & Non & Non \\
\hline \multirow{4}{*}{\multicolumn{2}{|c|}{ 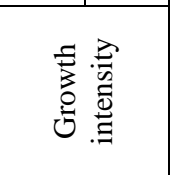 }} & REB5 & ++ & +++ & + & + & + & \pm & + \\
\hline & & REB9 & ++ & + & \pm & + & + & \pm & + \\
\hline & & G12 & + & \pm & + & + & + & \pm & \pm \\
\hline & & DEG18 & - & - & - & - & - & - & - \\
\hline
\end{tabular}




\begin{tabular}{|c|c|c|c|c|c|c|c|c|c|}
\hline \multirow{8}{*}{ 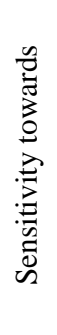 } & \multirow{4}{*}{$\begin{array}{l}\frac{\pi}{0} \\
\text { Z } \\
\text { Z }\end{array}$} & REB5 & - & - & - & - & - & - & - \\
\hline & & REB9 & - & - & - & - & - & - & - \\
\hline & & G12 & - & - & - & - & - & - & - \\
\hline & & DEG18 & - & - & - & - & - & - & - \\
\hline & \multirow{4}{*}{$\vec{U}$} & REB5 & - & - & - & - & - & - & - \\
\hline & & REB9 & - & - & - & + & + & - & - \\
\hline & & G12 & - & - & - & - & - & - & - \\
\hline & & DEG18 & - & - & - & - & - & - & - \\
\hline
\end{tabular}

- no growth, \pm doubt growth, + growth, ++ moderate growth, and +++ heavy growth.

Table 4. Physiological and chemo-type characterization for the selected Streptomyces isolates.

\begin{tabular}{|c|c|c|c|c|c|}
\hline & Items & $\begin{array}{l}\text { Streptomyces sp. } \\
\text { strain REB5 }\end{array}$ & $\begin{array}{c}\text { Streptomyces canaries } \\
\text { strain REB9 }\end{array}$ & $\begin{array}{c}\text { Streptomyces } \\
\text { sp. } \\
\text { strain G12 } \\
\end{array}$ & $\begin{array}{c}\text { Streptomyces } \\
\text { albus } \\
\text { strain DEG18 }\end{array}$ \\
\hline \multirow{2}{*}{$\begin{array}{c}\text { Chemotaxo } \\
\text { nomic } \\
\text { characteris } \\
\text { tics } \\
\end{array}$} & $\begin{array}{r}\text { Whole cell } \\
\text { hydrolysate }\end{array}$ & LL-DAP & LL-DAP & LL-DAP & LL-DAP \\
\hline & $\begin{array}{l}\text { Whole cell sugar } \\
\text { pattern }\end{array}$ & $\begin{array}{c}\text { Non characteristic } \\
\text { sugar (glucose) }\end{array}$ & $\begin{array}{l}\text { Non characteristic } \\
\text { sugar (glucose) }\end{array}$ & $\begin{array}{c}\text { Non } \\
\text { characteristic } \\
\text { sugar } \\
\end{array}$ & $\begin{array}{l}\text { Galactose \& } \\
\text { glucose }\end{array}$ \\
\hline \multirow{34}{*}{$\begin{array}{l}\text { Physiologic } \\
\text { al } \\
\text { characteris } \\
\text { tics }\end{array}$} & $\begin{array}{c}\text { Liquefaction of } \\
\text { gelatin }\end{array}$ & + & - & 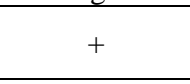 & + \\
\hline & $\begin{array}{c}\text { Coagulation of } \\
\text { milk }\end{array}$ & + & + & + & + \\
\hline & $\begin{array}{l}\text { Hydrolysis of } \\
\text { starch }\end{array}$ & \pm & - & - & - \\
\hline & $\begin{array}{l}\text { Decomposition of } \\
\text { cellulose }\end{array}$ & + & ++ & + & ++ \\
\hline & Melanoid pigment & - & - & - & - \\
\hline & \multicolumn{5}{|c|}{ Carbon utilization } \\
\hline & Glucose & + & + & +++ & ++ \\
\hline & Fructose & + & - & + & + \\
\hline & Galactose & ++ & + & + & - \\
\hline & Xylose & + & + & ++ & + \\
\hline & Rhamnose & ++ & + & ++ & + \\
\hline & Lactose & + & + & + & +++ \\
\hline & Sucrose & + & + & ++ & +++ \\
\hline & Maltose & + & + & + & + \\
\hline & Starch & ++ & + & ++ & + \\
\hline & Cellulose & - & +++ & + & +++ \\
\hline & Sodium acetate & + & ++ & + & - \\
\hline & Sodium citrat & + & + & - & - \\
\hline & Mannitol & +++ & ++ & + & - \\
\hline & Arabinose & ++ & ++ & + & + \\
\hline & Raffinose & - & +++ & - & - \\
\hline & Inositol & + & +++ & - & - \\
\hline & \multicolumn{5}{|c|}{ Nitrogen utilization } \\
\hline & L- proline & +++ & +++ & +++ & ++ \\
\hline & L-methionine & + & ++ & + & + \\
\hline & DL-phenylalanine & +++ & ++ & ++ & + \\
\hline & L-histidine & +++ & ++ & ++ & + \\
\hline & Peptone & +++ & + & +++ & + \\
\hline & Tryptone & ++ & + & +++ & + \\
\hline & L-tyrosine & +++ & ++ & +++ & ++ \\
\hline & Sodium nitrate & ++ & ++ & \pm & + \\
\hline & Casein & ++ & ++ & ++ & + \\
\hline & $\begin{array}{l}\text { Ammonium } \\
\text { sulphate }\end{array}$ & ++ & ++ & + & ++ \\
\hline & Salt tolerance & $\leq 60 \mathrm{~g} / 1$ & $\leq 60 \mathrm{~g} / 1$ & $\leq 70 \mathrm{~g} / 1$ & $\leq 60 \mathrm{~g} / 1$ \\
\hline
\end{tabular}

- no growth, \pm doubt growth, + growth, ++ moderate growth, and +++ heavy growth.

$\leq 60 \mathrm{~g} / \mathrm{l}$ : growth occurred till concentration of salt $\leq 60 \mathrm{~g} / \mathrm{l}$

$\leq 70 \mathrm{~g} / 1$ : growth occurred till concentration of salt $\leq 70 \mathrm{~g} / 1$ 


\section{Bioactivity of streptomycetes species in secondary screening}

In secondary screening, all of these streptomycetes were detected to have the ability to inhibit the growth of one or another tested pathogens. The findings in the present study had exceeded the estimation of Ndonde and Semu (2000), where about $75 \%$ of Streptomyces species were estimated to produce antimicrobial substances of one type or another. This indicates that the potential of these marine environments was sampled to harbor antimicrobial-producing Streptomyces species. Preliminary data in Table 5 showed that all the streptomycetes species tested were inhibitory to at least one Gram-positive bacterium and one Gram-negative bacterium. All of them inhibited growth of Enterobacter cloacae. On the other hand, four strains inhibited at least one of the tested fungi and inhibited growth of Fusarium oxysporum. In secondary screening, growth inhibition of tested Gram-positive bacteria decreased by $25.8 \%$ of bioactivity in primary screening, while growth inhibition of tested Gram-negative bacteria decreased by $6.2 \%$; no streptomycetes species showed any activity against Klebsiella pneumoniae. It could be suggested that a higher concentration of bioactive metabolites inhibitory towards Gram-positive and Gram-negative bacteria were produced in solid culture (Tan et al., 2004). On the other hand, growth inhibition of fungi was increased by $6 \%$, growth inhibition of Alternaria alternata was evident in secondary screening, but none in primary screening. This indicated that the diffusible extracellular metabolites in solid medium did not induce this antifungal activity.

Extraction with ethyl acetate and acetone were important for the production of antifungal compounds from Streptomyces albus strain DEG18 against Fusarium oxysporum and Alternaria alternata in secondary screening, while it was none in primary screening. Extraction with ethyl acetate and acetone were not suitable for the same strain to produce antibacterial activities. In addition, these extractions were not suitable for all strains to produce potent activity against Enterobacter cloacae. The solvents were used for extraction may not be suitable for the strains (Pandey et al., 2002). This was contrary to the results reported by Farida et al., (2007); Lin and Liu, (2010); Attimarad et al., (2012); Jose et al., (2013); Bundale et al., (2015) who found that ethyl acetate is the most appropriate solvents for antibiotic extraction. This might be due to presence of greater amount of active antimicrobial components which are more soluble in organic solvent than water (Karima et al., 2015). All selected strains of Streptomyces species displayed a broad spectrum activity against at least one of the fungi, Gram-positive and Gram-negative bacteria tested in secondary screening. Bioactivity of a single strain of Streptomyces against a variety of pathogenic microorganisms indicated that a single strain of Streptomyces could possibly produce a variety of antimicrobial substances. These strains could possibly produce the same bioactive metabolites inhibitory against the Grampositive and Gram-negative bacteria tested. Many antibacterial compounds have the inhibitory effect against both of Gram-positive and Gram-negative bacteria and produced previously from Streptomyces violaceus and Streptomyces coelicolor (Hobbs et al., 1992); Streptomyces tokumonensis (Betina, 1994) and Streptomyces tenebrarius H6 (Du et al., 2004).

Comparison of antibacterial bioactivity of marinederived actinomycetes in primary and secondary screenings revealed that all strains were active against at least the same one tested bacterium in both primary and secondary screenings. The same pattern of activity indicated that the Streptomyces spp. produced extracellular and intracellular bioactive metabolites antagonistic towards the same microorganisms. Interestingly, Streptomyces albus strain DEG18 that was inactive against $F$. oxysporum and Alternaria alternata in primary screening, inhibited them in secondary screening (Table 5). There were a few factors that could lead to this pattern of improved activity. Probably, the low concentration of the bioactive metabolites or the intracellularly-bound bioactive metabolites within the Streptomyces species was the reason why no inhibition was detected in primary screening. In addition, the increased production of the intracellular or extracellular bioactive metabolites in liquid medium and subsequently in the crude extracts might have increased the antifungal potential of the strains in secondary screening (Tan, 2007). The extraction of the intracellular or membrane-bound bioactive metabolites needed to be performed on this strain. Fragmentation of mycelia in liquid medium during fermentation might cause inactivation of the bioactive metabolites in the extracts (Shomura $e t$ al., 1979; Tan, 2007). Thus, this could explain the non-inhibitory effect of some strains Streptomyces species against some tested bacteria and fungi in secondary screening, although they were 
inhibitory towards them in primary screening. The insufficient bioactive metabolites in the crude extracts do not reach the effective dose could be another possible reason for the non-inhibitory effect (Tan, 2007). Streptomyces albus strain

Table 5. Metabolites and biomass extraction antimicrobial activity for the selected Streptomyces strains against some bacteria and fungi as inhibition zone $(\mathrm{mm})$. Data represented as means of three replications with standard error.

\begin{tabular}{|c|c|c|c|c|c|c|c|c|c|c|}
\hline \multirow{2}{*}{$\begin{array}{l}\text { Strain name and } \\
\text { fractions }\end{array}$} & \multicolumn{6}{|c|}{ Tested Bacteria } & \multicolumn{4}{|c|}{ Tested fungi } \\
\hline & $\begin{array}{c}E . \\
\text { cloacae }\end{array}$ & \begin{tabular}{|c|} 
K. \\
penumoniae
\end{tabular} & $\begin{array}{c}E \\
\text { coli }\end{array}$ & $\begin{array}{c}S . \\
\text { aureus }\end{array}$ & $\begin{array}{c}\text { B. } \\
\text { cereus }\end{array}$ & $\begin{array}{c}B . \\
\text { subtilis }\end{array}$ & $\begin{array}{c}F . \\
\text { oxysporium }\end{array}$ & \begin{tabular}{|c|} 
A. \\
niger
\end{tabular} & \begin{tabular}{|c|} 
A. \\
flavus
\end{tabular} & $\begin{array}{c}A . \\
\text { alternata }\end{array}$ \\
\hline \multirow{3}{*}{$\begin{array}{c}\text { Streptomyces sp. } \\
\text { strain REB5 }\end{array}$} & $0.6 \pm 0.0$ & \begin{tabular}{|l|}
0 \\
\end{tabular} & $3.3 \pm 0.0$ & $3.18 \pm 0.5$ & $4.0 \pm 0.3$ & 0 & $1.35 \pm 0.35$ & 0 & 0 & 0 \\
\hline & 0 & 0 & 0 & 0 & 0 & 0 & $1.05 \pm 0.05$ & 0 & 0 & 0 \\
\hline & 0 & 0 & 0 & 0 & $3.05 \pm 0.05$ & 0 & 0 & 0 & 0 & 0 \\
\hline \multirow{3}{*}{$\begin{array}{c}\text { Streptomyces canaries } \\
\text { strain REB9 }\end{array}$} & $2.55 \pm 0.25$ & 0 & 0 & $5.35 \pm 0.05$ & $2.47 \pm 0.37$ & 0 & $4.35 \pm 0.05$ & 0 & 0 & 0 \\
\hline & 0 & 0 & 0 & 0 & 0 & 0 & 3 & 0 & 0 & 0 \\
\hline & 0 & 0 & 0 & 0 & 0 & 0 & 0 & 0 & 0 & 0 \\
\hline \multirow{3}{*}{$\begin{array}{l}\text { Streptomyces sp. } \\
\text { strain G12 }\end{array}$} & 4.8 & 0 & 0 & 0 & 0 & $6.49 \pm 0.19$ & $5.5 \pm 0.09$ & 0 & 0 & 0 \\
\hline & 0 & 0 & 0 & 0 & 0 & 0 & $4.9 \pm 0.09$ & 0 & 0 & 0 \\
\hline & 0 & 0 & 0 & 0 & 0 & 0 & $8.5 \pm 0.5$ & 0 & 0 & 0 \\
\hline \multirow[t]{3}{*}{$\begin{array}{l}\text { Streptomyces albus } \\
\text { strain DEG18 }\end{array}$} & $1.19 \pm 0.015$ & 0 & 0 & 0 & $4 \pm 1.01$ & $1.15 \pm 0.15$ & 0 & 0 & 0 & 0 \\
\hline & 0 & 0 & 0 & 0 & $2.35 \pm 0.05$ & 0 & $4.05 \pm 0.04$ & 0 & 0 & 0 \\
\hline & 0 & 0 & 0 & 0 & 0 & 0 & 0 & 0 & 0 & $9.2 \pm 0.2$ \\
\hline
\end{tabular}

Deepa et al. (2013) found that, all the sixteen actinomycete isolates comprised Streptomyces albus were highly active against Staphylococcus aureus and Klebsiella pneumoniae. Pandey et al. (2002) found that 27 out of $36(75 \%)$ and 31 $(86.1 \%)$ actinomycetes were active against $S$. aureus and B. subtilis, respectively in primary screening. In secondary screening, 23 out of 36 (63.9\%) strains were inhibitory towards both $B$. subtilis and S. aureus. Zheng et al. (2000) reported that $B$. subtilis was inhibited by nine out of fifteen Streptomyces species with an inhibition zone diameter of less than $10 \mathrm{~mm}$. Enterobacter cloacae was described as the most susceptible bacterial species because all Streptomyces species showed antibacterial activity against it in both primary and secondary screenings. These results showed that diffusible extracellular metabolites produced on agar plate and the intracellular or extracellular metabolites in liquid medium and subsequently in the crude extracts could greatly induce the antibacterial activity against Enterobacter cloacae. Aspergillus niger was the most insensitive microorganism, where all Streptomyces species were inactive toward it in both primary and secondary screenings, this indicated that these strains did not produce intracellular and extracellular bioactive metabolites inhibitory towards it (Tables 2 and 5).
DEG18 and Streptomyces sp strain REB5 were promising strains for the production of bioactive metabolites with suitable solvents.
This was contrary to the results reported by Deepa et al. (2013) and Nandhini et al. (2015), who isolated different strains of Streptomyces albus from South East Coast of India and Tamil Nadu coastal areas, respectively, which showed the maximum level of inhibition zone towards the Aspergillus niger. According to Ndonde and Semu (2000), the sensitivity of the pathogens tested to the bioactive metabolites produced by the Streptomyces species might due to non-exposure of the pathogens tested to similar bioactive metabolites previously. As a result, they were still susceptible to such metabolites. Greater resistance of the pathogens tested might be due to previous exposure to antibiotics routinely used in disease control which might be similar to those produced by the present Streptomyces species. In addition, the sensitivity of the antimicrobial substances $e x$ situ towards light and temperature, the natural instability after prolonged storage, or low amount of the bioactive substances present in the crude extracts were the possible explanations for the low antimicrobial potential (Tan et al., 2004).

\section{Conclusion}

In this study, four marine Streptomyces strains ( $S$. albus DEG18, S. canaries REB9, $S$. sp. REB5 and 
S. sp. G12) were isolated and identified based on the morphological, chemotaxonomic and physiological characterizations. The primary screening for those strains showed a wide range of antimicrobial activities. Extractions from their metabolites using ethyl acetate and their biomass using acetone exhibited also antimicrobial activities toward some bacteria and fungi. The bioactive metabolites from those marine Streptomyces strains are promising for probable novel antimicrobial agents' production. This would need more structural characterizations in the future work.

\section{References}

Adegboye MF, Babalola OO (2013). Actinomycetes: a yet in exhaustive source of bioactive secondary metabolites. In: Microbial pathogens and strategies for combating them: Science, Technology and Education (Mendez-vilas, A.Ed.); pp: 786-795.

Alan TB, James SMS (2007). Marine action- bacteria; new opportunities for natural product search and discovery. Microbiol; 15: 491-499.

Anderson AS, Wellington MHE (2001). The taxonomy of Streptomyces and related genera. Int J Syst Evol Microbiol; 51: 797-814.

Attimarad SL, Ediga GN, Karigar AA, Karadi R, Chandrashekhar N, Chandrashekara S (2012). Screening, isolation and purification of antibacterial agents from marine actinomycetes. Int Curr Pharm J; 1(12): 394-402.

Ballav S, Kerkar S, Thomas S, Augustine N (2015). Halophilic and halotolerant actinomycetes from a marine saltern of Gao, India producing antibacterial metabolites. J Biosci Bioeng; 119(3): 323330.

Becker B, Lechevalier MP, Gordon RE, Lechevalier HA (1964). Rapid differentiation between Nocardia and Streptomyces by paper chromatography of whole-cell Hydrolysate. Appl Microbiol; 12: 421423.

Becker B, Lechevalier MP, Lechevalier HA (1965). Chemical composition of cell-wall preparations from strains of various form-genera of aerobic actinomycetes. Appl Microbiol; 13: 236-243.

Berdy J (2005). Bioactive microbial metabolites. J Antibiot; 58: 1-26.

Betina V (1994). Bioactive Secondary Metabolites of Microorganisms. Amsterdam: Elsevier.

Bouvier T, del Giorgio PA (2007). Key role of selective viral- induced mortality in determining marine bacterial community composition. Environ Microbiol; 9: 287-297.
Bredholdt H, Fjaervik E, Johnsen G, Zotchev SB (2008). Actinomycetes from sediments in the Trondheim fjord, Norway: diversity and biological activity. Mar Drugs; 23:12-24.

Bull AT, Ward AC, Goodfellow M (2000). Search and discovery strategies for biotechnology: the paradigm shift. Microbiol Mol Biol Rev; 64: 573606.

Bundale S, Begde D, Nashikkar N, Kadam T (2015). Optimization of culture conditions for production of bioactive metabolites by Streptomyces spp. isolated from soil. Advances in Microb; 5: 441-451.

Carte BK. (1996). Biomedical potential of marine natural products. Biosciences; 46: 271-86.

Das S, Lyla PS, Khan SA (2006). Marine microbial diversity and ecology: importance and future perspectives. Curr Sci; 90:1325-1335.

Deepa S, Kanimozhi K, Panneerselvam A (2013). 16rDNA phylogenetic analysis of actinomycetes isolated from marine environment associated antimicrobial activities. Hygeia J D Med; 5 (2): 43 50.

Du Y, Li T, Wang YG, Xia H (2004). Identification and functional analysis of dTDP Glucose-4,6Dehydratase gene cluster in an aminoglycoside antibiotics producer of Streptomyces tenebrarius H6. Curr Microb; 49: 99-107.

Ellaiah P, Reddy APC (1987). Isolation of actinomycetes from marine sediments of Visakhapatnam, east coast of India. Indian J Mar Sci; 16: 134-135.

Farida Y, Widada J, Meiyanto E (2007). Combination methods for screening marine actinomycetes producing potential compounds as anticancer. Indonesian J Biotechn; 12(2): 988-997.

Feling RH, Buchanan GO, Mincer TJ, Kauffman CA, Jensen PR, Fenical W (2003). Salinosporamide A: a highly cytotoxic proteasome inhibitor from a novel microbial source, a marine bacterium of the new genus Salinospora. Angew Chem Int Ed Engl; 42: 355-357.

Fenical W, Jensen PR (2006). Developing a new resource for drug discovery: marine actinomycete bacteria. Nat Chem Biol; 2: 666-667.

Gontang EA, Fenical W, Jensen P R (2007). Phylogenetic diversity of gram- positive bacteria cultured from marine sediments. Appl. Environ. Microbiol; 73: 3272- 3282.

Guimarães LM, Furlan, RL, Garrido LM, Ventura A, Padilla G, Facciotti MC (2004). Effect of pH on the production of the antitumor antibiotic retamycin by Streptomyces olindensis. Biotechn Appl Biochem; 40:107-111.

Hames-Kocabas E, Uzel A (2012). Isolation strategies of marine- derived actinomycetes from sponge and sediment samples. J Microb Methods; 88: 342-347. 
Hobbs G, Obanye AIC, Petty J, Mason JC, Barratt E, Garner DCJ, Flett F, Smith CP, Broda P, Oliver SG (1992). An integrated approach to studying regulation of production of the antibiotic methylenomycin by Streptomyces coelicolor A3(2). J Bacteriol; 174: 1487-1494.

Hong K, Gao AH, Xie QY, Gao H, Zhuang L, Lin HP (2009). Actinomycetes for marine drug discovery isolated from mangrove soils and plants in China. Mar Drugs; 7: 24-44.

Hotam SC, Bhavana S, Anju RS, Sourabh S (2013). Diversity and versatility of Actinomycetes and its role in antibiotic production. J App Pharm Sci; 3: S83-S94.

Jensen PR, Gontang E, Mafnas C, Mincer TJ, Fenical W (2005). Culturable marine actinomycete diversity from tropical Pacific Ocean sediments. Environ Microbiol; 7:1039-1048.

Johnson IF, Curl EA, Bond JH, Fibourg HA (1959). Methods for studying soil microflora-plant disease relationships. Burgess publishing Co., Minneapolis, USA.

Jose PA, Sivakala KK, Jebakumar SRD (2013). Formulation and statistical optimization of culture medium for improved production of antimicrobial compound by Streptomyces sp. JAJ06. Int J Microbiol; Volume 2013, Article ID 526260, 1-10.

Junker B, Walker A, Hesse M, Lester M, Christensen J, Connors N (2009). Actinomycetes scale- up for the producing of the antibacterial, nocathiacin. Biotechn Prog; 25 (1): 176-188.

Karima S, Farida S, Mihoub ZM (2015). Antioxidant and antimicrobial activities Plantago major. Int $\mathbf{J}$ Pharm Pharm Sci; 7(5): 58-64.

Kavithambigai E (2006). Diversity and biological characteristic of actinomycetes associated with roots of Rhizosphora sp. Master Thesis, University of Malaya, Kuala Lumpur.

Kieser T, Bibb MJ, Buttner MJ, Chater KF (2000). Practical Streptomyces genetics. Hopwood D. A. (Ed.). John Innes Centre, Norwich, England.

Kijjoa A, Sawangwong P (2004). Drugs and cosmetics from the sea. Mar Drugs; 2: 73-82.

Koch E, Loffler L (2009). Partial characterization of the antimicrobial activity of Streptomyces antimycoticus FZB53. J Phytothology; 157 (4): 235-242

Lam KS (2006). Discovery of novel metabolites from marine actinomycetes. Curr Opin Microbiol; 9:245-251.

Lechevalier H, Lechevalier M P (1981). Introduction to the order Actinomycetales. In: Starr M P; Stolp H G; Balows A; Schlegel H G (Eds.). The Prokaryotes. Germany: Springer- Verlag, Berlin; 2: 1915-1922.

Lin QX, Liu Y (2010). A new marine microorganism strain L0809: Taxonomy and characterization of active compounds from its metabolites. World $\mathbf{J}$ Microbiol Biotechn; 26: 1549-1556.

Lyudmila A R, Masataka U, Natalia I K, Valery V M (2008). Isolation, phylogenetic analysis and screening of marine mollusc- associated bacteria for antimicrobial, hemolytic and surface activities. Microbiol Res; 163 (6): 633-664.

Maldonado LA, Stach JE, Pathom-aree W, Ward AC, Bull AT, Goodfellow M (2005). Diversity of cultivable actinobacteria in geographically widespread marine sediments. Antonie Van Leeuwenhoek; 87: 11-18.

Manivasagan P, Kang KH, Sivakumar K, Li-Chan ECY, Oh HM, Kim SK (2014). Marine actinobacteria: An important source of bioactive natural products. Environ Toxic Pharm; 38: 172188.

Nandhini SV, Bharathy PJ, Rekha S (2015). Antifungal compounds from marine Streptomyces. Int J Pharm Pharm Sci; 7(1): 207-209.

Ndonde MJM, Semu E (2000). Preliminary characterization of some Streptomyces species from four Tanzanian soils and their antimicrobial potential against selected plant and animal pathogenic bacteria. World J Microbiol Biotechn; 16: 595-599.

Pandey B, Ghimire P, Agrawal VP (2002). Studies on the antibacterial activity of the actinomycetes isolated from the Khumbu region of Nepal. Available online: (http://www.aehms.org/pdf/Panday\%20F.pdf). Assessed on 8 May 2006.

Pridham TG, Tresner HD (1974a). Family Streptomycetaceae Waksman and Henrici. pp.747 748. In: R.E. Buchanan and N.E. Gibbons (eds.), Bergey's manual of determinative bacteriology. 8th ed. The Williams and Wilkins Co., Baltimore.

Pridham TG, Tresner HD (1974b). Genus I. Streptomyces Waksman and Henrici. pp.748-829. In: R.E. Buchanan and N.E. Gibbons (eds.), Bergey's manual of determinative bacteriology. 8th ed. The Williams and Wilkins Co., Baltimore.

Qiu D, Ruan J, Huang Y (2008). Selective isolation and rapid identification of members of the genus Micromonospora. Appl Environ Microbiol; 74: 5593-5597.

Ramesh S, Mathivanan N (2009). Screening of marine actinomycetes isolated from the Bay of Bengal, India for antimicrobial activity and industrial enzymes. World J Microbiol Biotechn; 25: 21032111.

Ravenschlay K, Salam K, Pernthater J, Amann R (1999). High bacterial diversity in permanently cold marine sediments. Appl Environ Microbiol; 65: 3982-3989.

Shams M, Shahnavaz B, Ghazvini K, Valinasab T (2015). Screening of actinomycetes from Lipar area 
of Oman Sea to investigate the antibacterial compounds. Avicenna J Clin Microb Infect.; 2(1): e23621.

Shirling EB, Gottlieb D (1968a). Cooperative description of type cultures of Streptomyces. II. Species descriptions from the first study. Int J Syst Bacteriol; 18: 69-189.

Shirling EB, Gottlieb D (1968b). Cooperative description of type cultures of Streptomyces III. Additional species description from first and second studies. Int J Syst Bacteriol; 18: 279-392.

Shirling EB, Gottlieb D (1969). Cooperative description of type cultures of Streptomyces. IV. Species description from the second, third and fourth studies. Int J Syst Bacteriol; 19: 391-512.

Shirling EB, Gottlieb D (1972). Cooperative description of type strains of Streptomyces. V. Additional descriptions. Int J Syst Bacteriol; 22: 265-394.

Shomura T, Yoshida J, Amano S, Kojima M, Inouye S, Niida T (1979). Studies on Actinomycetales producing antibiotics only on agar culture. I. Screening, taxonomy and morphology productivity relationship of Streptomyces halstedii, strain SF1993. J Antibiotics; 32: 427-435.

Stach JE, Maldonado LA, Masson DC, Ward AC, Goodfellow M, Bull AT (2003). Statistical approaches for estimating actinobacterial diversity in marine sediments. Appl Environ Microbiol; 69: 6189-6200.

Stanek JL, Roberts GD (1974). Simplified approach to identification of aerobic actinomycetes by thin- layer chromatography. Appl Microbiol J; 28: 226231.

Tan CJ (2007). Biological and chemical characterization of actinomycetes isolated from selected marine macroorganisms from Peninsular Malaysia. Master Thesis, University of Malaya, Kuala Lumpur.

Tan CJ, Vikineswary S, Thong KL, Affendi YA (2004). Antagonistic activities of selected actinomycetes isolated from marine organisms against Candida albicans, C. parapsilosis and selected pathogenic fungus and bacteria. In: Phang et al. (Eds), Marine Science into the New Millennium: New Perspective and Challenges. (p. 489-495).

Williams ST, Cross T (1971). Actinomycetes isolation from soil, Methods in microbiology, Academic press, London, New York. 4: 295-334.

Williams ST, Sharpe ME, Holt JG (1989). Bergey's Manual of Systematic Bacteriology. Williams and Williams, Baltimore, London.

Wu RY (1984). Studies on the Streptomyces SC4. II. Taxonomic and biological characteristics of Streptomyces strain SC4. Bot Bull Acad Sci; 25: 111-123.

Zhang J, Zhang L (2011). Improvement of an isolation medium for actinomycetes. Mod Appl Sci; 5: 124127.

Zheng Z, Zeng W, Huang Y, Yang Z, Li J, Cai H, Su W (2000). Detection of antitumor and antimicrobial activities in marine organism associated actinomycetes isolated from the Taiwan Strait, China, FEMS Microbiology Letters; 188: 87-91.

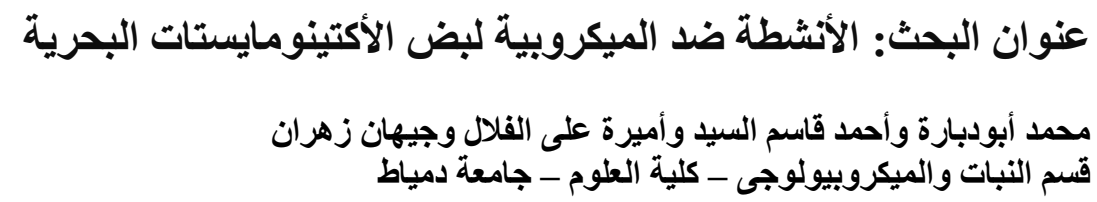

تم عزل عشرون عزلة أكتينومايستات بحرية من ساحل شمال دلتا مصر، كما تم اختيار و تعريف أربعة

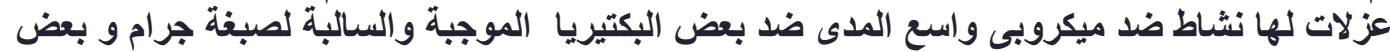
الفطريات، تم تعريف العزلات على أساس الصفات المات المورفولوجية و الفسيولوجية و الخواصية لصنية التصنيفية

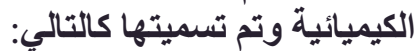

Streptomyces albus strain DEG18, Streptomyces canaries strain REB9, Streptomyces sp strain G12. 9Streptomyces sp. strain REB5

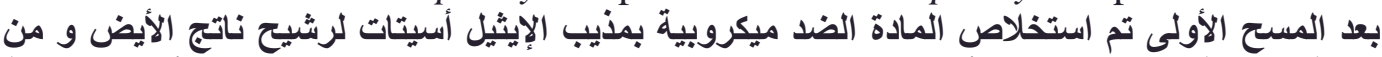

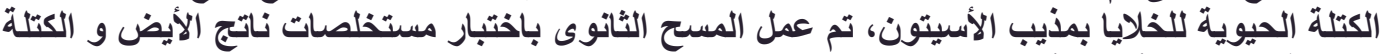

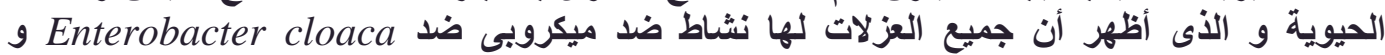

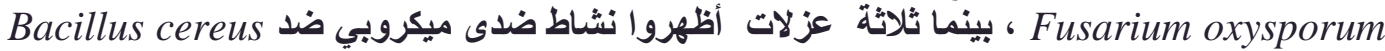

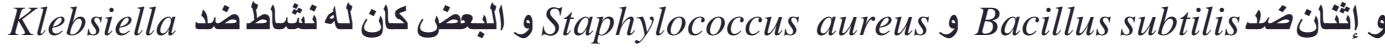
Alternaria alternata .pneumoniae 